\title{
El Islote de Santa Cruz, iMucho más Que un ObJeto DE INVESTIGACIÓN !..*
}

\author{
Por: Carmenza Jiménez Torrado \\ Diana Monroy Burgos \\ Estela Fuentes Fuentes \\ Alfonso Henriquez Narváez
}

\section{RESUMEN}

* Artículo producto de la investigación: La Configuración del Islote de Santa Cruz. 2003. Trabajo de grado, Especialización en Teorías, Métodos y Técnicas de Investigación Social Tutores de la Investigación: Orlando Durango Rueda e Isabel Pérez Chaín.

${ }^{1}$ ROSS, Marc Howard. La Cultura del Conflicto, las $\mathrm{D}$ i f e re n c i a s Interculturales en la Práctica de la Violencia. Buenos Aires. Argentina. Paidos. 1993

2 HENAO, Hernán y VILLEGAS, Lucelly. Estudios de Localidades, Módulo 5 de Investigación Social. ICFES: 1997

3 Todo lo que en adelante aparece entre comillas

son

$\mathrm{f} r$ a $\mathrm{g} \mathrm{m}$ e $\mathrm{nt}$ o s textualmente tomados de entrevistas o historias de vida aplicadas en la Investigación los cuales sirvieron como información primaria.

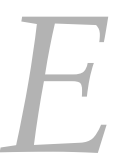

ste artículo representa un esfuerzo por mostrar algunos hallazgos que, a nuestro parecer, particulariza un grupo poblacional cuyas interacciones le aproximan estrechamente a lo que Ross ${ }^{1}$ llama vínculos cruzados, entendidos éstos como una red de relaciones atravesadas por sentimientos que facilitan el alcance de intereses comunes.

El diseño de Investigación, marco de las discusión que aquí se presenta, es un estudio de localidad; elección que hemos hecho porque entendemos que en la localidad se puede percibir mejor hasta donde se reconoce el propio territorio, y se da sentido y nombre a las personas, a los animales y las calles, se reconocen espacios significativos como los lugares geográficos, los monumentos y demás objetos reales o imaginarios que le dan sentido al lugar y a las relaciones entre sus pobladores, donde se admiten trazos de homogeneidad y heterogeneidad.

La localidad es el referente obligado, afirma Hernán $\mathrm{Henao}^{2}$, del individuo que pretende decir quién es, afirmarse en sus valores y concretar el intangible que se define como idiosincrasia. Es también el espacio donde las comunidades se miran y tienen conciencia de si mismas y donde se reconoce la diversidad cultural.

\section{...El Islote de Santa Cruz: Parece un Enjambre de Casas Colgantes sobre el Mar*}

"Los primeritos venían de Barú, los Cortés, ellos fueron los primeros, ellos construyeron sus primeros ranchos y permitieron que los demás construyeran; no le pedían permiso a nadie..." ${ }^{3}$ 
El anterior testimonio fue uno de los primeros ofrecidos por el señor Miguel Felipe Morelo, conocido como el Tío Pepe, el habitante más anciano y que no solo carga sobre sus hombros toda la historia oral de la isla, sino en gran parte la tranquilidad de la misma. Un hombre con 106 nietos y 70 bisnietos y que logró "la hazaña" de mantener una sana convivencia entre sus tres esposas, quienes actualmente aun viven tan pacíficamente desde cuando decidieron "compartir un mismo hombre".

Así es este pequeño arrecife, alejado de "todo mal", lleno de historias, con un clima tropical, cuya forma podría asemejarse irónicamente a la de una hoja, o

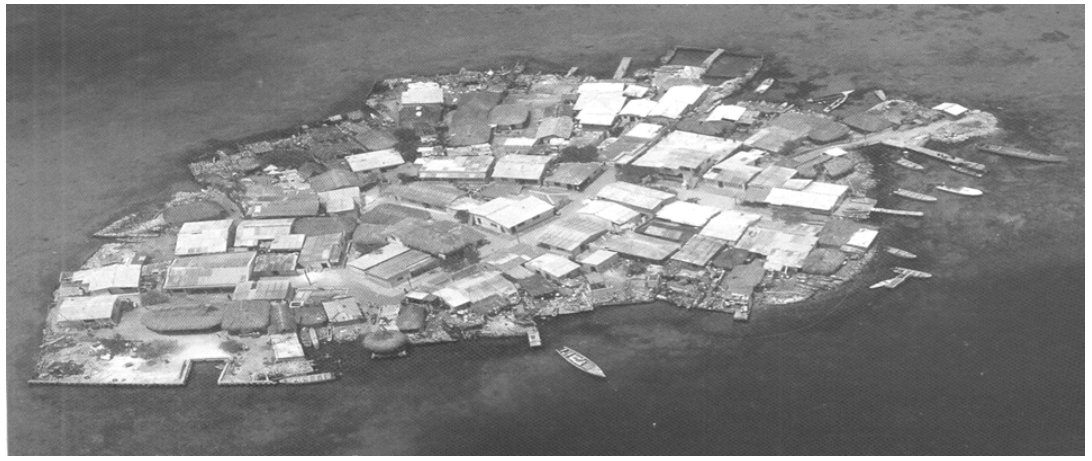
por pura coincidencia, a la de un pez; una comunidad "extraña", "única", "diferente" en palabras de muchos reporteros, visitantes o simples turistas que diariamente pasan por allí por ser camino obligado para llegar a las otras nueve (9) islas que componen el archipiélago de San Bernardo donde se encuentra ubicada, frente a las costas del mar Caribe, cerca al municipio de San Onofre y a una hora en lancha del municipio de Santiago de Tolú.

\section{iCómo Hace tanta Gente para Vivir en un Lugar tan Pequeño y Alejado de Todos y de Todo!...}

Al señor Felipe le llaman Tío Pepe no sólo por apodo, sino porque se trata casi de un hecho real, pues efectivamente es tío de casi todo el pueblo. Cuenta con ochenta y cinco (85) años y es orgullosamente descendiente directo del fundador del poblado, su bisabuelo Fermín Cortés, y de la "Cacica" como apodaron por mucho tiempo a su bisabuela, María Lucas Julio. Cuenta tío Pepe que por casi tres generaciones las mujeres fueron las que imponían el "orden" en la isla, prioridad que sigue vigente y que hoy es responsabilidad que comparte Tío Pepe con Doña Elena o la Abuela como la llaman. ${ }^{4}$

En el mapa levantado a mano alzada que incluimos aquí, se precisa el área física total del Islote de Santa Cruz, aproximadamente $8.340 \mathrm{~m}^{2}$, superficie cubierta en casi su totalidad por edificaciones que sirven de vivienda a los isleños. En éste, el $40 \%$ de las edificaciones se encuentran localizadas en la parte suroriental, donde se concentra la mayor parte de la población, el 13\% en la parte céntrica, el 21\% en el sector noroccidental, y el 26\% en la parte norte. Esta

${ }^{4}$ De tal manera que si se hacen cuentas aproximadas desde el momento en que el bisabuelo del señor Felipe decidió que la Isla iba a ser su lugar de residencia y el de su descendencia han pasado casi dos siglos de fundada. 
porción de la Isla fue el primer lugar en formarse por las manos de los pescadores que con coral, arena y desechos de coco fueron levantando primero casuchas y luego edificaciones de material. Fue aquí donde llegaron aquellos pescadores que venían detrás de las tortugas carey:

"No solo encontraron las tortugas, sino también encontraron un sitio que aunque inhóspito para algunos, fue para los pescadores conocedores de la región el lugar perfecto, pues -no habían bichos como en las otras islas... fue descubrir una tierra prometida como descubrir a América como sucedió con los españoles-" ${ }^{5}$.

Las creencias en el islote se tejen en torno a un Ser Supremo que los protege contra los peligros de quien les brinda, "hogar, comida y hasta tranquilidad": el mar. Es que, paradójicamente, el mayor temor de los santacruceros es que ese mismo mar les arrebate todo lo que les brinda. Su afán por evidenciar su apego religioso va desde el nombre mismo de su isla, Santa Cruz, pasando por la nomenclatura popular de cada una de sus calles, Calle de Dios, de la Cruz, de Santa Rita, entre otras. "El mar no nos ha mostrado su hondo"(...) "nos ha dejado seguir construyendo "afirma

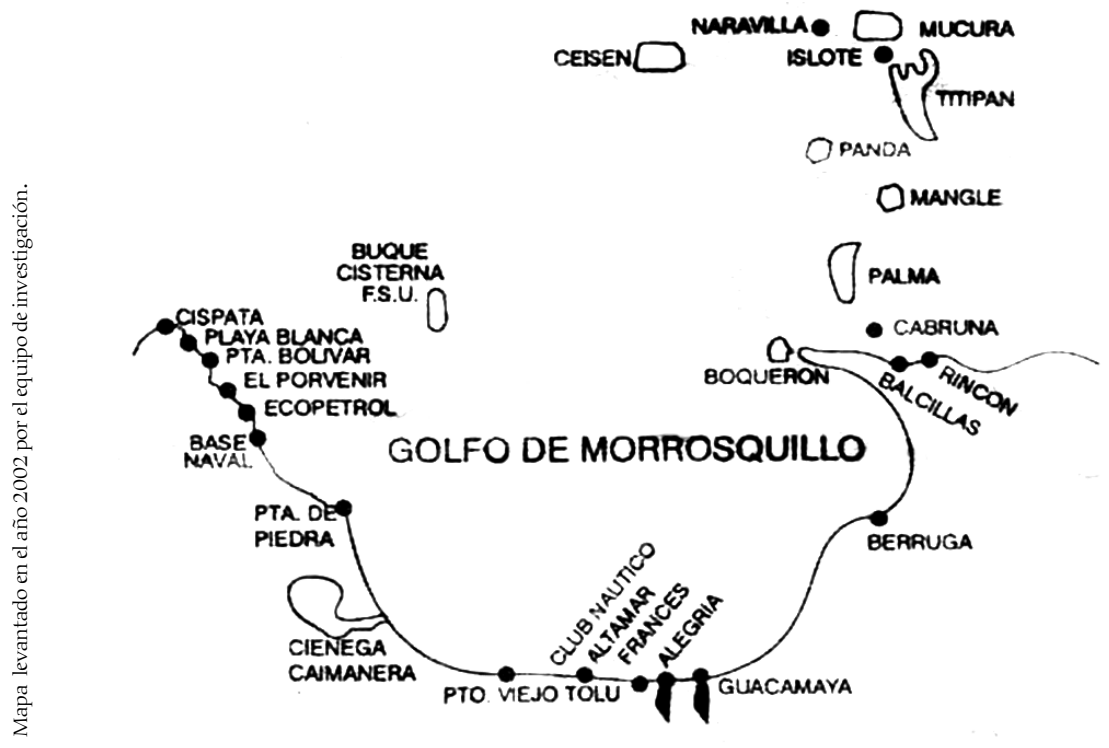
Hidalgo un anciano de ochenta y seis (86) años quien ha vivido casi toda su vida en la Isla "me trajeron cuando era un niño y aquí me quedé", Para él como para el resto de sus habitantes el mar ha sido muy benévolo con ellos $\mathrm{y}$, sobre todo, Dios que "lo es todo". "Lastima", dice Susana, "que aquí no hay un párroco permanente, hay que traerlo de Tolú, cuando hay bautizos, matrimonios o una muerte, esas celebraciones las respetamos mucho aunque no tengamos iglesia". Y Doña Elena comenta que "eso se vive en cada uno; pero cumplimos, creemos, respetamos". Lo ideológico en esta comunidad irrumpe y se queda en los demás sistemas que conforman la dinámica social y cultural del Islote.

${ }^{5}$ Entrevista a Miguel Felipe Morelo, Isla de Santa Cruz, 2000

\section{¿Qué Pasa con los Espacios en el Islote?...}

Insistimos que la localidad es algo más que un conjunto de ladrillos; es la 
expresión visible de un sistema de valores ligados a las creencias, ideas y esperanzas del hombre y la mujer. Cada cultura tiene su forma particular de cultivar la tierra, de construir sus templos, de organizar su poblado y vías de comunicación, de elegir sus lugares de celebraciones y fiestas. Y del mismo modo, cada persona tiene su forma particular de organizar su vivienda, y sus espacios, creando instancias o rincones que van adquiriendo valor para ella.

Los descendientes de las primeras familias "fueron consiguiendo mujer entonces había que construir", afirma Tío Pepe, "las calles son verdaderos laberintos en donde se entretejen no solo la estructura física sino las relaciones sociales de la Isla".

Para los isleños lo público no es sólo el espacio en el que coinciden las personas, ni tampoco un espacio de consumo y producción; es mucho más que eso, o más bien, es diferente a eso. En la Isla existen lugares. Y los lugares no son un espacio cualquiera, son espacios con significado, y éste significado se lo dan ellos y ellas todo el tiempo.

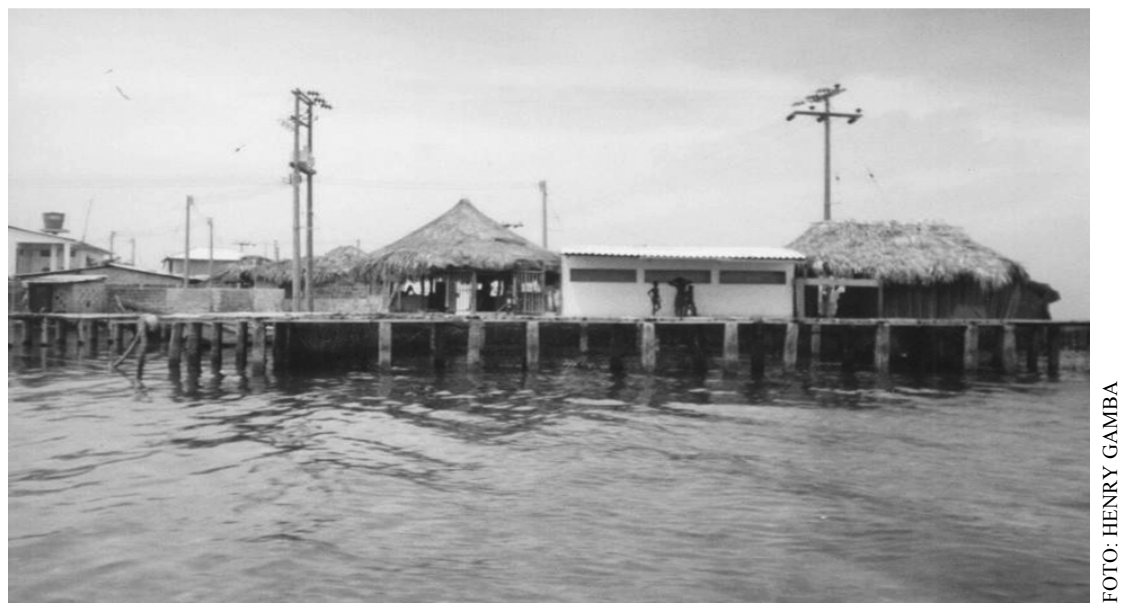

Dice Tío Pepe que cada quien iba agarrando su pedazo de tierra e iba construyendo su casa, sus chozas al principio, "y si tenia que traer pedazos de coco o escombro lo traía, todos nos ayudamos (...), no cualquiera, tampoco, eran los familiares de los primeros, los amigos, los compañeros de pesca. Tenía que ser buena gente; debe ser buena gente la que puede construir aquí. Si usted quiere ahora construir ya es difícil, ya no hay espacio, pero si es amigo se queda en la casa de cualquiera (...); aquí los nativos nos casamos con los nativos, es muy difícil que consigamos gente afuera, eso trae mucho problema (...), yo a mis hijos les iba construyendo, esta es toda una cuadra de mis hijos; ahí atrás en el solar iba parando su casa el hijo mayor y luego el otro y el otro, y así vamos construyendo entre más cerquita mejor, así nos ayudamos; usted nos ve así, patio con patio, pero no hay problema, al contrario(...), siempre me llaman para que les de consejos sobre construcción, todos me llaman así sea para que les mire nada más. Es que Tío Pepe sí sabe donde construir, dice la gente (...), yo sé dónde el mar muestra su hondo, eso hay que respetarlo, y no todo el mundo sabe eso, además mi todavía me respetan porque yo doy ejemplo". 6

El puerto, por ejemplo, uno de los más representativos lugares, para los isleños 
tiene "dueño": doña "Elena", que por haber traído unas bolsas de arena y cemento y construido el muelle ya es legítima su propiedad; pero, al tiempo, el muelle es el "lugar de todos", de reuniones de bohemios en las horas de la noche, de jóvenes y de adultos en el atardecer, haciendo de este lugar un espacio en la que se cumplen importantes procesos de socialización.

El adulto mayor cuenta sus historias de conquista, escuchadas por los más jóvenes que las hacen suyas, historias de grandes hazañas en la pesca o historias sorprendentes de la forma como se construyó la Isla. Quién fue el primero que llego a la Isla, dónde se ubicó, con quién llegó. Todas las conversaciones tienen diversas y claras intenciones; por un lado, preservar la historia de la Isla y, por otro, reafirmar el sentido de pertenencia del territorio, "porque no fue nada fácil para los bisabuelos del Tío Pepe empezar a construir, eso no fue fácil, les tocó muy duro, ellos solos a los Cortez Julio, la primera familia que llego a la Isla... (...) Fermín Cortez y su esposa, son recordados tanto por los mayores como por los más jóvenes ya que aparte de ser los bisabuelos del Tío Pepe son las raíces consanguíneas de casi todos los que viven allí. El arraigo y la identificación que experimentan los habitantes del islote se traduce en una sensación de seguridad. El lugar les ampara, pues las personas han vertido en él su trabajo, sus ideas, lo han convertido, en cierto modo, en una prolongación de sí mismos.

No importa cuanto espacio público haya, lo importante es que, poco o mucho, éste se convierta en un lugar legitimado por quienes lo habitan. Es así como el muelle, la plaza de la cruz o la pequeña cancha, cada esquina, cada estrecha calle, cobran significado en la medida, como dice Tío pepe, "que son construidas con el sudor de nuestra frente, a nuestro gusto, es una extensión de nosotros mismo".

Para el isleño esta es otra posibilidad de entenderse más que como simples vecinos, pues lo hacen como miembros de una misma familia. Las terrazas, los patios, las estrechas calles, también son sitios de encuentros donde se discuten y se resuelven públicamente todo tipo de aspectos desde los más cotidianos hasta los más complejos entre ellos los conflictos.

Parecería que vivir tan cerca los unos de los otros y las otras podría ser un detonante que generase un sinnúmero de dificultades, pero no es así: dice el Señor Julio que

“(...) allá en la ciudad, aunque también viven en esos apartamentos y esos edificios, en esas cajas de fósforos, tan cerquita como aquí, allá hay un problema: ni se conocen, no se tienen confianza, mandan a poner esas rejas, tan altas para que ni se saluden. 
Aquí no, usted ve y todos nos conocemos, nos tenemos confianza, usted puede amanecer en cualquier calle, y no se le pierde na', así con joyas, y no le hace falta na' (...)

Esta cercanía que va más allá de estar conviviendo físicamente tan cerca, ha traspasado sin duda estas barreras; la cercanía les ha generado la posibilidad de construir, en palabras de Ross, ${ }^{7}$ una poderosa red de vínculos de construcción, vínculos afectivos que no solo toca éste campo sino que trasciende a la esfera de lo económico, lo político y social. Esto se puede explicar mejor si citamos la teoría de los vínculos cruzados, la cual plantea que las sociedades que tienen este tipo de vínculos desarrollan con más facilidad un poderoso y positivo sentido grupal y de intereses comunes, que aquellas sociedades que poseen vínculos reforzantes. Cuando las alianzas y otras modalidades de cooperación son fáciles de establecer con la vecindad, Leine y Campbell (1972), comentan que tales grupos manifiestan con frecuencia mayores niveles de etnocentrismo, lo que hace que se comporten más celosamente cuando se enfrentan a los extraños.

La teoría de los vínculos cruzados que plantea Roos intenta resaltar de qué forma la fuerza y la configuración de los lazos económicos, sociales, políticos y afectivos entre miembros de una sociedad influyen en el origen y en la expansión de los conflictos y hacen que sea más o menos factible su resolución pacifica (Coleman, 1957, Gluckman, 1995 y Colson, 1953). Si existen vínculos reforzantes, el círculo familiar, de vecindad, de edad, de religión y las afiliaciones políticas, colocan a la persona en un grupo central de gente que participa de esos roles. En cambio, si existen vínculos cruzados el individuo participa de distintas afiliaciones en gente diferente.

Aquí debemos recurrir a entender el proceso socioafectivo de construcción de la "socialidad" ${ }^{8}$, la cual tiene que ver en parte por la construcción de vínculos. La socialidad viene determinada por unas cálidas y afectuosas relaciones sociales que proporcionan al niño, que luego se convierte en hombre o mujer adultos, unos lazos con los demás que le dan seguridad y que paradójicamente facilitan el proceso de su separación e individualización, haciendo que la pérdida del objeto sea menos temida y facilitando asimismo la unión con los demás (Trevarthen y Logotheti, 1989).

Lo público es el producto de acuerdos comunes, de lo comunicado y validado en la sociedad por los particulares, y hace referencia a un patrimonio social. En consecuencia, construir lo público es dialogar, incluir, someter a discusión, buscar transparencia, validar, etcétera. De tal manera que la resolución de conflictos puede asumirse como un evento público en la medida que los demás (y esto tiene

7 Op.Cit. ROSS, Marc Howard. La Cultura del Conflicto, las D i f e r e n c i a $\mathrm{s}$ Interculturales en la Práctica de la Violencia. Buenos Aires. Argentina. Paidos. 1993

${ }^{8}$ Termino equivalente al de sociabilidad, aunque con un significado más amplio que describe las c a r a c terís tic a s i n d i vid u a l e s predominantes que conducen a la cooperación con los demás. 
9 Aún no se tiene precisión de la población total de la Isla, ya que los distintos censos realizados pueden mostrar un margen de error al momento de contabilizar la población. Se tiene conocimiento de que el último censo realizado en temporada vacacional arrojó una población mayor que la censada en esta investigación citada, ya que en él se tuvo en cuenta las personas que se encontraban viviendo por cuestiones de estudio y trabajo en sitios como Cartagena y otras zonas de los Departamentos de Bolívar y Sucre, los cuales estaban de regreso en la Isla. que ver con que los familiares y los vecinos, los otros y las otras, puedan intervenir en su resolución.

De nuevo aplicado al caso de Santa Cruz, podemos afirmar, sin temor a equivocarnos, que además de los vínculos consanguíneos y filiales se generan vínculos de pertenencia a un grupo especifico. Para el caso de los habitantes de la isla, estos vínculos traspasan la frontera de lo privado entendiendo como grupo a todos los habitantes con los que construyen esos objetivos comunes que no solo tienen que ver con su propia supervivencia sino también con la protección, el afecto y la sana convivencia.

\section{Son Tantas Personas...}

Según el censo realizado en el año 2002 por el equipo de Investigación, son 553 personas viviendo y conviviendo en $8.340 \mathrm{mts}^{2}$, casi en su totalidad ocupado por viviendas 82 en total, en las cuales habitan 126 familias, 69 de ellas habitan 25 viviendas $^{9}$. (Ver Tabla $\mathrm{N}^{\circ} 1$ )

Tabla No.1

Datos sobre Población

\begin{tabular}{|l|c|c|}
\hline Datos Poblacionales & CANTIDAD & PorCENTAJE \\
\hline Total Población & 553 habitantes & $100 \%$ \\
\hline Total hombres & 283 habitantes & $51 \%$ \\
\hline Total mujeres & 270 habitantes & $49 \%$ \\
\hline Número de familias & 126 & $100 \%$ \\
\hline Número de viviendas & 82 & $100 \%$ \\
\hline
\end{tabular}

Fuente: Censo realizado por el grupo de investigación. Noviembre de 2002

El total de la población frente al número de familias, genera un promedio de 4,3 miembros por familia y, a su vez, la distribución promedio de estas personas por vivienda es de 7 miembros, aproximadamente.

Confrontando la información de la población actual censada (553), número de familias (126) y número de viviendas (82); 44 familias, que equivalen al 34,92\% del total de la población, no cuentan con vivienda, sólo el 65,08\% de las familias las poseen. No obstante, 25 viviendas se encuentran habitadas por 69 familias, que equivalen al 55\%, las 57 viviendas restantes las ocupan 57 familias, es decir, el 45\%. (Ver tabla No. 2). 
Tabla No. 2

Hábitat Poblacional

HÁBITAT POBLACIONAL

\begin{tabular}{|l|l|}
\hline En 1 vivienda viven 5 familias & (5 familias en una sola vivienda) \\
\hline En 4 viviendas viven 15 familias & (4 familias por vivienda) \\
\hline En 8 viviendas viven 24 familias & (3 familias por vivienda) \\
\hline En 12 viviendas viven 24 familias & (2 familias por vivienda) \\
\hline En 57 viviendas viven 57 familias & (1 familia por vivienda) \\
\hline
\end{tabular}

Fuente: Censo realizado por el grupo de investigación. Noviembre de 2002

\section{Población y Edades}

El 55\% de la población se encuentra en edades entre 0 y 20 años, incluidos los bebés, niños y niñas, preadolescentes, adolescentes y jóvenes menores de edad; el $20 \%$ del total de la población se encuentra en una edad intermedia, que oscila entre 21 y 30 años; el $22 \%$ de la población, posee una edad que oscila entre 31 y 60 años; mientras que el 3\% restante son considerados los adultos mayores o personas de la tercera edad. (Ver Tabla $\mathrm{N}^{\circ}$ 3)

Tabla No. 3

\begin{tabular}{|l|l|}
\hline \multicolumn{2}{|c|}{ Rango de Edades } \\
\hline \multicolumn{2}{|c|}{ RANGOS De EDADES } \\
\hline De 0 a 5 años & 85 personas \\
\hline De 6 a 10 años & 75 personas \\
\hline De 11 a 15 años & 57 personas \\
\hline De 16 a 20 años & 60 personas \\
\hline De 21 a 25 años & 56 personas \\
\hline De 26 a 30 años & 56 personas \\
\hline De 31 a 40 años & 56 personas \\
\hline De 41 a 50 años & 35 personas \\
\hline De 51 a 60 años & 33 personas \\
\hline De 61 a más años & 16 personas \\
\hline TotAL & 529 Personas \\
\hline
\end{tabular}

Fuente: Censo realizado por el grupo de investigación. Noviembre de 2002

La alta densidad poblacional de la isla tiene que ver no sólo con su crecimiento vegetativo (277 personas de 529 tiene menos de 20 años), sino con la longevidad de los isleños. Es corriente encontrar entre los Santa Cruceros ancianos de más de 85 años, con un grado de participación colectiva importante. 
Tabla No. 4

Personas con Mayor Edad que aun Viven

\begin{tabular}{|l|l|}
\hline \multicolumn{2}{|l|}{ PERSONAS CON MAYOR EDAD QUE NACIERON Y ViVEN EN LA ISLA } \\
\hline Miguel Morelo & 85 años \\
\hline Señora de Miguel & 79 años \\
\hline Señora Elena (del Restaurante) & 73 años \\
\hline Ayda Castillo & 72 años \\
\hline Elida Castillo & 72 años \\
\hline Patricia Quintero & 71 años \\
\hline Esther Libia & 70 años \\
\hline
\end{tabular}

Fuente: Censo realizado por el grupo de investigación. Noviembre de 2002

Se estima, por los rangos de edades presentados, que en el mediano, corto y largo plazo, el 55\% de la población en edad menor de 20 años tiende a reproducirse, lo que puede generar un alto impacto en el crecimiento poblacional de la Isla, razón que llevará sin duda a que los nativos sigan ampliando terreno, ya que la alternativa de irse a vivir a otro lugar es casi remota para los Santa Cruceros, sobre todo del adulto «sabemos que vamos a seguir creciendo y reproduciéndonos, pero afortunadamente, ya hay otros que aprendieron a buscar el hondo al mar, ya saben como construir, de donde traer el material, mis hijos ya saben, seguiremos ampliando... es mejor estar aquí donde hay de todo, que allá afuera hay mucha maldad"10.

\section{Los Compadres son Sagrados}

La anterior información alarmaría a cualquier estudio sobre hacinamiento, pero para los Isleños convivir en familias numerosas es casi una tradición cultural que comparten como constante con las familias afro-descendientes. Interesa hacer una precisión frente a la información planteada. En primer lugar, por familia estamos entendiendo aquel conjunto de miembros unidos por vínculos de parentesco, que comparten un apellido; con ésta definición sencilla, precisada así por los autores de este artículo, se hizo el censo anterior. Haciendo la salvedad que para los Isleños es otra la compresión que tienen de los que es familia. Para ellos, familia son todos los que habitan en la isla, unos y otros son entendidos como familia, Evidentemente allí existe un vinculo consanguíneo, pero para los isleños el concepto es enriquecido por el valor que tienen de amistad; así el amigo

${ }^{10}$ Entrevista a Miguel Felipe Morelo, Isla de Santa Cruz, 2000. o amiga es familia pues comparte el mismo espacio y organiza conjuntamente la supervivencia. 
Entender la familia en este contexto es aceptar que estamos frente a una institución con características diferentes a lo que los teóricos de familia definen como tal, empezando por el número de miembros que la conforman; la familia en la Isla de Santa Cruz comprende diferentes parejas y diferentes miembros unidos fundamentalmente por la consanguinidad; comprende al abuelo, la abuela, tíos, padres, nietos, primos, hijos de crianza y algunos amigos y amigas y hasta el compadre.

Escoger al padrino para el hijo o la hija es otro ritual en la Isla ya que lo que se hace es escoger un padre sustituto, y también a una persona que servirá de mediador en caso de conflictos familiares. El padrino es una persona legitimada socialmente por cumplir con este doble papel:

"Aquí se respeta mucho a los compadres, después que uno pone un padrino es pá respétalo, ya uno le habla con mucho respeto, ellos también cuando ven a uno discutiendo con alguien se pueden meter y calmar la cosa (...) Se les respeta mucho, ellos le salvan a uno al hijo de la garras del Mohan, vuelven al hijo persona con el bautismo. Por eso se respetan tanto." 11

El compadre entra a ser parte de la parentela, y en ocasiones ya lo es de antemano, lo que refuerza aún más los lazos entra padres biológicos y compadres, al extremo de llegar a "sacralizarse" estos enlaces: "Los compadres son sagrados... son como una segunda persona después de la madre." 12

Es de mayor relevancia en la vida del Isleño la figura del compadre a la de la comadre, lo que puede estar ligado al hecho de que el hombre es autoridad y representa apoyo económico para la red y se espera entonces que cumpla con ésta función es en ausencia temporal o definitiva del padre biológico del niño o de la niña.

\section{Para Concluir sin Concluir...}

Queremos concluir este artículo retomando lo que se planteó al comienzo. Tiene que ver con que existen muchas pruebas (entre estas la comunidad del Islote de Santa Cruz) de que los seres humanos están predispuestos desde su nacimiento a establecer lazos sociales y a que las fuertes ataduras con otras personas tienen significación adaptativa. Volcán (1988) opina que la forma en la que esta esencial tarea se lleve acabo es parte integrante de un proceso universal basado

en la definición de aliados y enemigos: o sea, de amigos y adversarios. Las

\footnotetext{
${ }^{11}$ Entrevista a Susana, Isla Santa Cruz, 2000.

12 Entrevista a Palindo, Isla Santa Cruz, 2000.
} 
experiencias externas le proporcionan a la persona la materia prima para la construcción de su mundo interno, el cual contendrá los modelos primordiales de interacción humana relativos al afecto, al conflicto, a la autoridad, al poder, a la comunidad y a otros conceptos que a lo largo de la vida sean relevantes.

Estas disposiciones son configuraciones internas que tiene significación para la acción externa. Dentro de la esfera misma del conflicto, necesitamos conocer no solo quiénes son los aliados y quienes los enemigos, sino también cómo se espera que se comporten y cuales son las acciones adecuadas que hay que tomar con ellos.

A pesar que en este articulo se pretendió mostrar como la cercanía física entre los isleños va más allá de lo meramente espacial y accede a niveles profundos de la conducta individual y social, también encontramos evidencias que este mismo hecho tiene que ver con las formas particulares como se asumen y encaran los conflictos.

\section{BIBLIOGRAFIA}

AGAR, M. H. Hacia un Lenguaje Etnográfico. En: GEERTZ, C. et al. El Surgimiento de la Antropología Postmoderna. Barcelona, España: Gedisa, 1992. p. 117-140

AVRUCH, Kevin. La Cultura y el Conflicto. En: AVRUCH, Kevin, BLACK, Peter W. y JOSEPH A. Conflicto y Resolución: Perspectivas Culturales. Nueva York, U. S. A.: Greenwood Press, 1991.

DELGADO, Hazel. Nueva Visión de la Cultura del Conflicto- Cultura del Conflicto. Documento de Internet.

GEERTZ, C. Descripción Densa: Hacia una Teoría Interpretativa de la Cultura. En: La Interpretación de las Culturas. Barcelona, España: Gedisa, 1994. p. 19-40

—. La InTerpretación de la Cultura. Editorial Gedisa, 2000

GOTTEZ, J. P. y LECOMPTE, M. D. Etnografía y Diseño Caritativo en Investigación Educativa. Madrid, España: Morata, 1988

HENAO Hernán y otros. Estudios de Localidades. ICFES: 1997. Modulo de Investigación Social.

RITZER, G. Teoría Sociológica Moderna. McGraw Hill Editores, 2002

ROSS, Marc Howard. La Cultura del Conflicto, las Diferencias Interculturales en la Práctica de la Violencia. Buenos Aires, Argentina: Paidos, 1993

—. La Cultura del Conflicto. Barcelona, España: Ediciones Paidos, 1995 
SPRADLEY, J. P. y McCURDY, D. W. La Experiencia Cultural: Etnografía en Sociedades Complejas. California/Tennesse, U. S. A., 1972

TYLER, S. A. La Etnografía Postmoderna: de Documento de lo Oculto a Documento Oculto. En: GEERTZ, C et al. El Surgimiento de la Antropología Postmoderna. Barcelona, España: Gedisa, 1992. p. 171-213

\section{BIOGRAFIA}

\section{CARMENZA JIMÉNEZ TORRADO}

Trabajadora Social. Universidad de Cartagena. Especialista en Teorías, Métodos y Técnicas de Investigación Social Universidad de Cartagena. Candidata a Maestría en estudios de Género, Mujer y Desarrollo. Universidad Nacional-Universidad de Cartagena.

\section{DIANA MONROY BURGOS}

Trabajadora Social. Universidad de Cartagena.

\section{ESTELA FUENTES FUENTES}

Licenciado en Pedagogía Reeducativa. Especialista en Educación Personalizada. Universidad de Manizales. Especialista en Teorías Métodos y Técnicas de Investigación Social. Universidad de Cartagena.

\section{ALFONSO HENRIQUEZ NARVAEZ}

Licenciado en Pedagogía Reeducativa. Especialista en Educación Personalizada. Universidad de Manizales. Especialista en Teorías Métodos y Técnicas de Investigación Social. Universidad de Cartagena. Magíster en Desarrollo Social. Universidad del Norte. 